\title{
Specification of multiple geomagnetic responses to variable solar wind and IMF input
}

\author{
Shing F. Fung ${ }^{1}$ and Xi Shao ${ }^{2}$ \\ ${ }^{1}$ Heliospheric Physics Laboratory, NASA Goddard Space Flight Center, Greenbelt MD 20771, USA \\ ${ }^{2}$ Department of Astronomy, University of Maryland College Park, MD, 20742, USA
}

Received: 21 November 2006 - Revised: 13 December 2007 - Accepted: 18 December 2007 - Published: 26 March 2008

\begin{abstract}
This paper shows that the state of the magnetosphere, resulting from continuous but variable forcing of the solar wind and the interplanetary magnetic field (IMF), can be empirically specified by a magnetospheric state vector $\boldsymbol{\Psi}$, consisting of a set of hourly-averaged magnetospheric driver and response parameters. It is demonstrated that there exists a correspondence between the magnetospheric driver and multiple geomagnetic response parameters. This parameter correspondence allows different magnetopsheric states to be specified by means of a look-up table, provided that the relative time lags between various driver (e.g. $V_{s w}$, IMF) and response parameters (e.g. $K_{p}, D_{s t}$, and $A E$ ) are taken into account. Using the magnetospheric state specifications, multiscale geomagnetic responses can then be simultaneously prescribed statistically from their corresponding driver parameters. Magnetospheric state specifications have been determined by using magnetospheric state parameter data taken in 1970-2000. Their validities have been tested by specifying the multi-geomagnetic responses over three representative intervals: (1) a magnetic cloud event, (2) a period of multiple storms, and (3) the years of 2001 and 2002. For all the intervals, we have found good correlation (with $r>0.75$ ) between the prescribed and observed geomagnetic indices at hourly resolution, and the magnetospheric state specifications are thus validated.
\end{abstract}

Keywords. Magnetosphere (Magnetospheric configuration and dynamics; Solar wind-magnetosphere interactions; Storms and substorms)

\section{Introduction}

Solar wind-magnetosphere interaction causes the compression of the Earth's magnetic field on the dayside and the for-

Correspondence to: Shing F. Fung

(shing.f.fung@nasa.gov) mation of the long magnetotail on the nightside. This general shape of the magnetosphere is maintained by the solar wind and interplanetary magnetic field (IMF), but it changes dynamically in response to the variable external forcing. In addition, the lower magnetospheric boundary is coupled to the ionosphere and atmosphere below, which are also directly affected by solar radiation and pre-existing conditions. Consequently, it is not easy to model the global magnetospheric dynamics without simultaneously accounting for the multiple processes affecting different parts of the magnetosphere. Understanding the interplay between the solar wind, IMF, magnetosphere, ionosphere, and the atmosphere is the essence of Sun-Earth Connection science, and will provide the basis for space weather predictions.

It is well known that the magnetosphere responds to both magnetic (Dungey, 1961) and viscous (Axford and Hines, 1961) interactions with the IMF and solar wind plasmas. Such interactions can lead to global-scale magnetospheric phenomena, such as geomagnetic storms and substorms, resulting in spectacular auroral displays and enhancements of space current systems, ionospheric conductivities and the radiation belts. The apparent direct responses of the magnetosphere to actions of external drivers strongly indicate the causal relationships between the drivers and responses, even though we still have not fully understood the detailed physics of the solar wind-magnetosphere interactions.

An interesting question arises, however, as to whether the magnetosphere (and ionosphere) plays an active role in determining its responses to the external drivers. For example, ionization and neutral wind structures in the ionosphere and atmosphere are directly affected by solar radiation, which is, by and large, independent of solar wind and IMF variations. The resulting magnetospheric boundary at low altitude would then have an effect on how the magnetosphere may respond to a given solar wind or IMF input. Along this vein, Opgenoorth et al. (1996), for example, pointed out that global magnetospheric state might determine the details of

Published by Copernicus Publications on behalf of the European Geosciences Union. 
the later substorm development. The primary challenge in space weather prediction then is to determine the magnetospheric response for a given set of magnetospheric input.

Traditional linear prediction filter techniques based on single input-output relations (Clauer, 1986; McPherron et al., 1988; Baker et al., 1990; Vassiliadis et al., 1995) have widely been used to forecast a particular magnetospheric response to a given input driver. These techniques, however, are too simplistic to prescribe the global magnetospheric state because they invariably ignore any underlying effects due to other parameters having different time lags. Even in the case when two input parameters are considered, such as solar wind speed $V_{s w}$ and IMF $B_{z}$, they are first combined to form a single electric field parameter before consideration is made for predicting a single geomagnetic response of interest. Such treatment would have neglected the effects of different input parameters that may actually have different geoeffective time scales. Vassiliadis et al. (2005) have clearly demonstrated the need of using multiple parameters to specify the global state of the magnetosphere.

In this paper we investigate the characterization of a magnetospheric state, which may be viewed as an encapsulation of the conditions or configuration of the magnetosphere resulting from both the drivers (external and internal) and responses of the magnetosphere. Although it is tempting to consider magnetospheric responses as the results of a given solar wind or IMF input, as is usually done in impulseresponse or prediction-filter modeling analyses (e.g. Clauer, 1986; Vassiliadis et al., 1995; Klimas et al., 1998) and other heuristic modeling efforts (e.g. Temerin and Li, 2002), we consider the multiple geomagnetic responses (e.g. $K_{p}, D_{s t}$, and $A E$ ) themselves as a part of the magnetospheric state characterization. This distinction is subtle but important because it allows the possibility of multiple drivers affecting a given response, and its feedback on the system.

In the following sections, we give a description of the magnetospheric state paradigm and a way to specify different magnetospheric state prescriptions. We will then validate those prescriptions by using them to prescribe simultaneously the multi-responses of the magnetosphere corresponding to the observed solar wind input during different intervals of interest.

\section{The magnetospheric state paradigm}

The changing solar wind and IMF conditions can result in a multitude of complex, multi-scale, magnetospheric dynamic phenomena or processes. The term "magnetospheric state" then refers to the nearly instantaneous global magnetospheric configuration resulting from those processes and conditions imposed by the solar wind and IMF. For example, Hoffman et al. (1988), Gussenhoven (1988) and Watanabe et al. (1998) investigated the possible magnetospheric ground state when the magnetosphere has experienced a long period of quies- cence. According to Gussenhoven (1988), the criteria for a magnetospheric ground state are: (1) the solar wind speed, $V_{s w}<\sim 400 \mathrm{~km} \mathrm{~s}^{-1}$, (2) the magnitude of the IMF $B_{z}$ (in Solar Magnetospheric coordinates) is $<2 \mathrm{nT}$, (3) the total magnitude of IMF is $<5 \mathrm{nT}$, and (4) all three conditions above are maintained for at least $2-4 \mathrm{~h}$. It is quite clear from this "ground state" example that adequate specification of a magnetospheric state will require the concomitance of multiple parameter conditions. The last condition suggests that the magnetosphere may have a memory time of about a few hours.

The magnetosphere is in a disturbed or excited state during a geomagnetic storm or substorm, when the magnetosphere goes through a sequence of transitions and configuration changes associated with energy storing, dissipation and recovery. An earlier study by Vassiliadis et al. (1995) described the magnetospheric state only in terms of geomagnetic responses. As we intend to show in this paper, magnetosphere states must be characterized by combinations of solar wind, IMF and the multi-geomagnetic response parameters (Fung, 1996; Fung et al., 2005).

Early attempts have been made (e.g. McPherron, 1974; Klimas et al., 1992) to characterize the state of the magnetosphere in terms of dayside reconnection magnetic flux, tail lobe open flux and return flux. Newell et al. (2001) also suggested using in addition state variables, such as the polar cap magnetic flux and a magnetotail stretching index, to parameterize the state of the magnetosphere's magnetic field. The disadvantage of these variables is that they are not routinely available and they do not account for the different actions of the solar wind and IMF input, which may lead to multiregional (e.g. magnetotail, ring current, and inner magnetosphere) processes that can cause more than just changes in the amounts of polar cap flux or stretching of the magnetotail.

As recognized by Newell et al. (2001) and others, the main distinction between different magnetospheric states is in the magnetospheric magnetic field configuration. This seems reasonable because the geomagnetic or magnetospheric magnetic field tends to play a role in most, if not all, magnetospheric processes. Therefore, in order to adequately prescribe a magnetospheric state, it is important to capture as completely as possible all the factors controlling the multiregional variations of the geomagnetic field due to solar wind and IMF input, and the ensuing geomagnetic responses, as proposed in Fung (1996). Using a straightforward successive localization process, we will describe next the specification technique that is able to account for the multivariate dependencies of magnetospheric states.

\section{Specification of magnetospheric states}

As suggested in Fung (1996), a magnetospheric state may be prescribed by both the input driver and the multiple 
geomagnetic response parameters. The collection of parameters then forms a "magnetospheric state vector" $\boldsymbol{\Psi}$, which as an example can be represented by

$$
\boldsymbol{\Psi}=\left[\boldsymbol{B}_{\mathrm{IMF}}, P_{S W}, F 10.7 ; K_{P}, D_{s t}, A E, A L ; \boldsymbol{\tau}\right]
$$

The first three parameters in Eq. (1) are the driver (input) parameters of magnetospheric processes, while $K_{P}, D_{s t}, A E$ and $A L$ are the multiple geomagnetic response parameters that have been nearly continuously monitored for several decades. In order to account for finite response times of different magnetospheric processes (global geomagnetic activity, ring current, and auroral currents), a lag-time vector $\boldsymbol{\tau}$ is introduced to specify the relative time shifts or effective histories of the different magnetospheric state parameters. The probability of occurrence of a given magnetopsheric state is thus given by the joint occurrence probability of the corresponding multi-parameter ranges.

Among the driver parameters in Eq. (1), the interplanetary magnetic field vector $\boldsymbol{B}_{\mathrm{IMF}}$ symbolically represents any combination of the field components $\left(B_{x}, B_{y}, B_{z}\right)$ and the total field magnitude $B_{\text {tot }}$. The inclusion of $B_{\text {tot }}$ in $\Psi$ is to account for its contribution to the total solar wind pressure exerted on the magnetosphere, particularly due to shock passages preceding magnetic clouds (Burlaga et al., 1990; Gosling, 1990). Similarly, $P_{S W}$ is shorthand for either the solar wind dynamic pressure, or the solar wind density $n_{s w}$ and speed $V_{s w}$, which may separately have different geo-effective consequences (e.g. see Fung and Tan, 1998). The F10.7 radio flux is a known solar activity indicator and thus may be used as a proxy to the solar UV flux that directly affects the ionosphere and atmosphere, i.e. the lower magnetospheric boundary.

On the other hand, magnetospheric responses involving different processes operating in different magnetospheric regions are complex and have different time and spatial scales. These processes (and their effects) may be conveniently and summarily represented in terms of the multiple geomagnetic indices, such as $K_{P}, D_{s t}, A E$, and $A L$ (Mayaud, 1980). Sampled at 3-hourly intervals globally, hourly at mid-latitudes, and on $\sim$ minute time scale at high latitudes, the $K_{p}, D_{s t}$, and auroral indices $(A E, A L)$, respectively, monitor different magnetospheric and ionospheric activities on different time scales in response to a given set of external driver conditions. Their inclusions in Eq. (1) are important for accounting for the effects of different response processes (such as ULF waves, ring current and ionospheric currents) in setting the state of the magnetosphere. In order to specify $\boldsymbol{\Psi}$ on an hourly time scale, we have processed the $K_{p}$ time series data into hourly resolution by extending the single $K_{p}$ value for a given 3-hourly interval into 3 hourly values to cover the same interval. In this way, $K_{p}$ would have the same resolution as the solar wind and other data. Vassiliadis et al. (2005) have shown recently that many of the state parameters in Eq. (1) affect different parts of the Earth's radiation belt.
It is important to recognize that although we have conveniently chosen to use $K_{P}, D_{s t}, A E$ and $A L$ for this study to represent the multiple geomagnetic responses, they are not unique for such a purpose and do not necessarily represent the optimal set of parameters needed to prescribe a magnetospheric state. The key point, though, is that both driver and response conditions are needed to specify the state of the magnetosphere. In the next sections, we examine the prescriptions of magnetospheric states in detail.

\section{Parameterization of a magnetospheric state}

Magnetospheric state (Eq. 1) is a multi-parameter empirical function that may depend nonlinearly on its parameters. Needless to say, the multiple geomagnetic responses are not mutually independent parameters as they are connected by yet to be identified cross-scale coupling processes in the global magnetic configuration.

To illustrate the complex relationship between a given magnetospheric response (e.g. $K_{p}$ ) and multiple driver (input) parameters, Fig. 1 shows the variations of $K_{p^{-}}$ distributions recorded in 1970-2003 as a function of different driver parameters: $V_{s w}, n_{s w}, P_{s w}, B_{\mathrm{tot}}$, and $B_{z}$, for the case of $\tau=0$. It is easy to see that the $K_{p}$-distribution changes with the variation of each of the driver parameters, and that the overall $K_{p}$ distributions are also different for different driver parameters (Fig. 1b-f). Suffice it to point out here that other geomagnetic response indices ( $D_{s t}$, and auroral index) behave similarly and their distributions are omitted here for brevity.

The prediction filter techniques that have been used extensively to investigate relationships of input-output parameter pairs tend to ignore the effects of multiple driver input on the variations of the output parameter; see panels (b-f) in Fig. 1 (e.g. Clauer, 1986; Vassiliadis et al., 1995). Recently, Vassiliadis et al. (2005) have shown that different magnetospheric state parameters can affect different magnetospheric regions. Therefore, the simultaneous consideration of multiple driver parameters and their corresponding multiple geomagnetic responses is necessary to properly specify the global state of the magnetosphere (Fung, 1996).

\subsection{Parameter localization}

Ukhorskiy et al. (2002) showed that multi-scale magnetopsheric responses may be handled by using multi-scale locallinear filters. More recently, Ukhorskiy et al. (2004) have also developed a relativistic-electron flux specification model for the geosynchronous region based on the development of single-output filter with multiple input parameters. These multi-scale local-linear filter techniques, like others mentioned earlier, can only produce one output parameter, which cannot be used to characterize the global state of the magnetosphere. A simpler way to ascertain the range combinations 


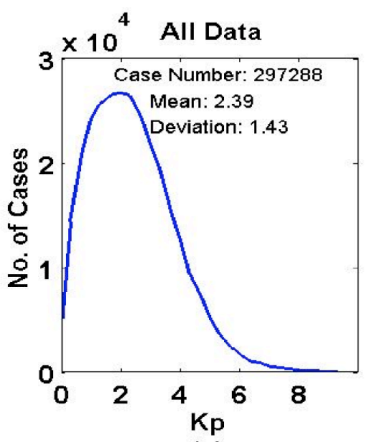

(a)

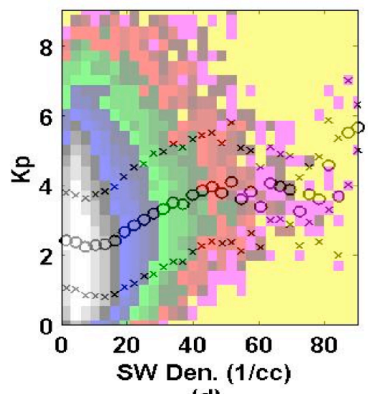

(d)

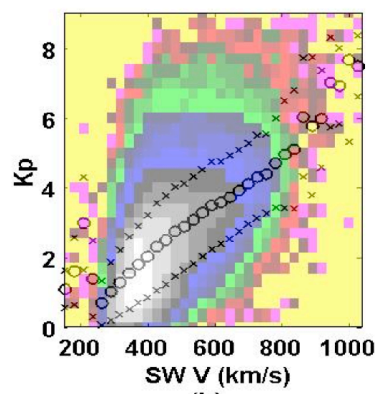

(b)

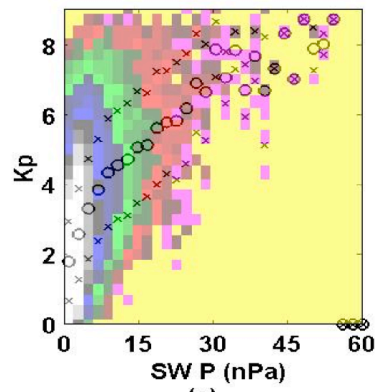

(e)

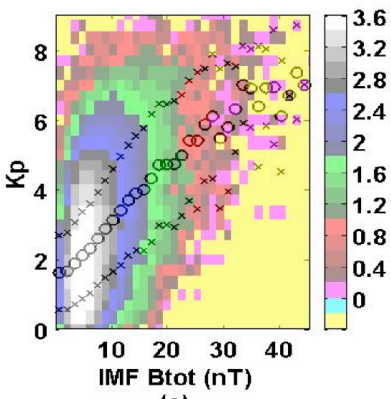

(c)

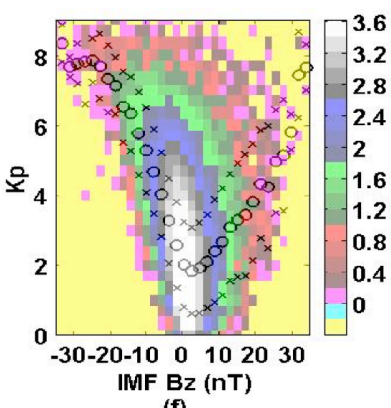

(f)

Fig. 1. Histogram of all 3-hourly $K_{p}$ index values (a) in 1970-2003, (b) as a function of solar wind speed $V_{s w}$, (c) as a function of IMF $B_{\text {tot }}$, (d) as a function of solar wind density $n_{s w}$, (e) as a function of solar wind dynamic pressure $P_{s w}$, and (f) as a function of IMF $B_{z}$. The color scale for panels (b-f) shows the logarithms of the numbers of occurrences. Open circles show the mean $K_{p}$ value while the crosses indicate $+/-$ a standard deviation. All the distributions, though rather broad, show monotonic variations of $K_{p}$ with hourly solar wind and $I M F$ input with zero time lag. We note that same $K_{p}$ values can result from either positive or negative IMF $B_{z}$.

of multiple parameters to characterize magnetospheric states, however, is to employ the widely used parameter-localization process, or binning (Duda et al., 2000; Theodoridis and Koutroumbas, 2003).

It is elementary to assert that the behavior of a multivariate function with respect to any variable of interest can always be investigated by holding all other independent variables constant or to specific small intervals. The variables or parameters that are held to specific intervals are then said to be localized. The variation of the function within each localizedparameter bin is assumed to be insignificant compared to the total change in the function with respect to the variable of interest. Therefore, the function's variability within the localized parameter bin and the average value of the parameter are then characteristic to that particular parameter bin. Since all magnetospheric-state parameters are treated similarly under localization, there is no preference in the order in which parameters are localized. Below, we will only use $K_{p}$ to demonstrate the parameter localization procedures.

As shown in panels (b-f) in Fig. 1 , while the mean $K_{p}$ (open circles) vary differently with each of the driver parameters, the spread of each of the $K_{p}$-distributions must be due to the underlying variations of all other (non-localized) parameters (drivers and responses) as well as random errors. In order to examine the true variation of a given response pa- rameter $\left(K_{p}, D_{s t}, A E\right.$, etc.) with another (driver or response) parameter, we need to localize all but the parameters in question.

To start the localization process, we have binned the full range of each of the dynamical driver parameters into five or six bins as follows (bin sizes and boundaries are only chosen as a matter of convenience):

$\left\{V_{s w, i}\left(\mathrm{~km} \mathrm{~s}^{-1}\right)\right\}=\{<400,400-500,500-600,600-750,>750\}$

$i=1,2,3,4,5$

$\left\{B_{\text {tot }, \mathrm{j}}(\mathrm{nT})\right\}=\{<5,5-10,10-15,15-25,>25\}$

$j=1,2,3,4,5$

$\left\{P_{s w, k}(\mathrm{nPa})\right\}=\{<5,5-10,10-15,15-20,>20\}$

$k=1,2,3,4,5$.

For every combination of these hydrodynamic parameter ranges, there can be a corresponding set of IMF conditions $\left\{B_{x, l}, B_{y, m}, B_{z, n}\right\}$ subject to the limitation of $B_{\mathrm{tot}, \mathrm{j}}$ with

$\left\{B_{x, l}(\mathrm{nT})\right\}=\{<-15,-15--5,-5-0,0-5,5-15,>15\}$

$l=1,2,3,4,5,6$

$\left\{B_{y, m}(\mathrm{nT})\right\}=\{<-15,-15-5,-5-0,0-5,5-15,>15\}$

$m=1,2,3,4,5,6$ 

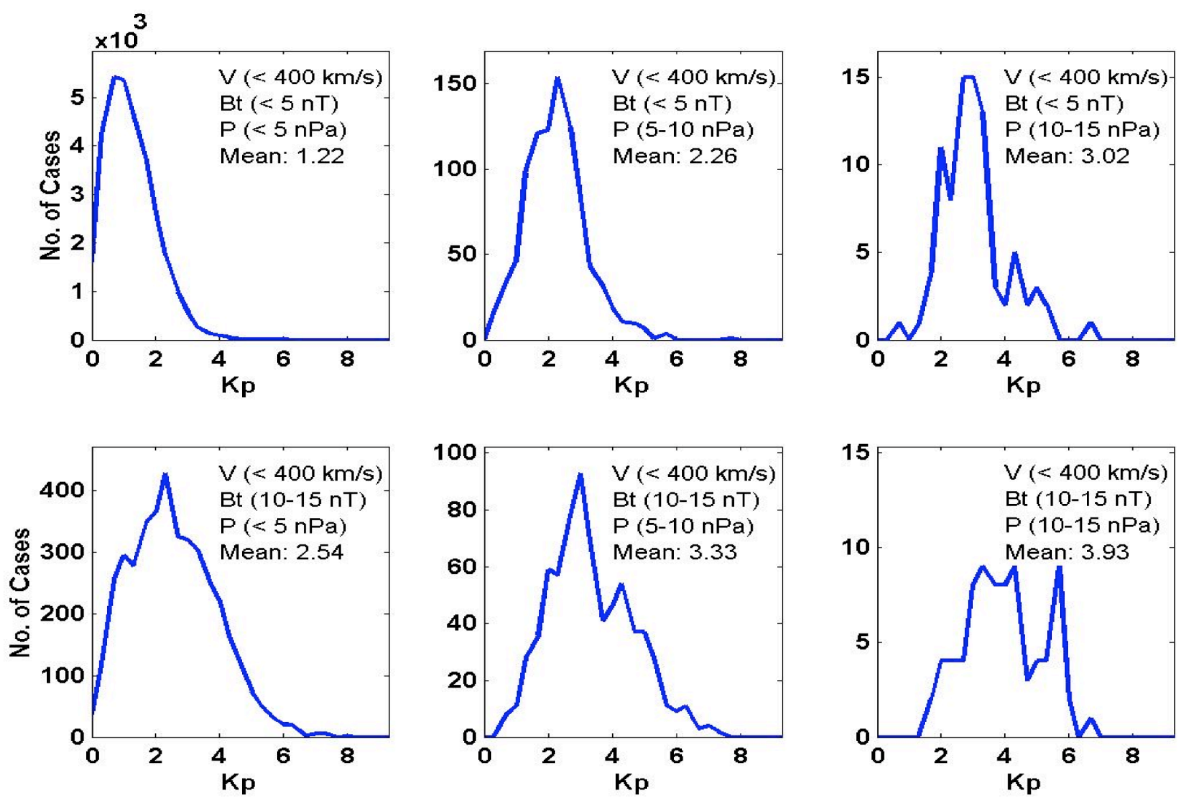

Fig. 2. Examples of reduced histograms of $K_{p}$ when $V_{s w}, P_{s w}$ and $B_{\text {tot }}$ are successively localized. In these examples, we have kept $V_{s w}<400 \mathrm{~km} \mathrm{~s}^{-1}$ while $P_{s w}$ increases from left $(<5 \mathrm{nPa})$ to right $(10-15 \mathrm{nPa})$ and the magnitude of IMF $B_{\text {tot }}$ increases from top $(<5 \mathrm{nT})$ to bottom (10-15 nT). We see that the peaks of the $K_{p}$ distributions shift to higher $K_{p}$ values as both $P_{s w}$ and $B_{\text {tot }}$ increase. In Figs. 1 and 2 , concurrent data are plotted.

$\left\{B_{z, n}(n T)\right\}=\{<-15,-15--5,-5-0,0-5,5-15,>15\}$

$n=1,2,3,4,5,6$.

With such binning, it is now possible to determine the true variation of a response parameter with respect to any driver parameter within each data bin, where all other state parameters are localized. In each localized bin combination then, the systematic variability of all other state-parameters are minimized, with only random error remaining. Different localized data bins also represent different parameter regimes with which the locally linear driver-response relationship may still vary due to nonlinearity. This process can be repeated for any pair of driver and response parameters in Eq. (1).

Figure 2 shows examples of different $K_{p}$-distributions when $V_{s w}, P_{s w}$, and $B_{\text {tot }}$ are successively localized. While the monotonic variations of the average $K_{p}$ with $P_{s w}$, and $B_{\text {tot }}$, as shown in Fig. 1 are preserved, the $K_{p}$ distributions in successively localized bins are defined with less uncertainty since they have narrower spreads than the non-localized distributions in Fig. 1.

In the current study, we have limited our considerations only to the following driver parameters: $V_{s w}, P_{s w}, B_{\text {tot }}$, and $B_{z}$ (ignoring $F 10.7$ for it has a much longer variability time scale). As shown in Fig. 1, all these parameters are essentially directly correlated with the average $K_{p}$ and thus are suitable for localization. Since changes in IMF $B_{y}$ and $B_{x}$ do not seem to yield significant variations in geomagnetic indices, we will omit them from the current study for simplicity. For $B_{z}$, however, different correlations exist for positive and negative $B_{z}$, suggesting that the same $K_{p}$ value can result from different interaction processes associated with the two IMF $B_{z}$ orientations. The solar wind density $n_{s w}$, which is contained in $P_{s w}$, is not considered separately because the average $K_{p}$ does not change significantly over the whole range of $n_{s w}$. In the following, we only use $K_{p}$ to demonstrate the localization process, although the same procedures are applicable to other state parameters.

It is important to note that parameter binning is a straightforward way to analyze a multivariate distribution function. As such, the complexity of a multi-parameter (multidimensional) problem that is otherwise difficult to be modeled from first principle (see, e.g. Sharma, 1995; Valdivia et al., 1996, 1999) can be treated easily. For example, different combinations of $V_{s w, i}$ and $B_{z, n}$ also handle the effects of the interplanetary electric field $E_{y, i, n}=V_{s w, i} B_{z, n}$.

An advantage of the multi-parameter localization or multivariate binning analysis is to enable the construction of the distributions of a response parameter (e.g. $\left.K_{p}\right)$ with respect to any chosen magnetospheric state parameter (e.g. $B_{\text {tot }}$ ), for different interval-averaged values (bins) of all other state variables (e.g. $B_{z}, V_{s w}, D_{s t}$, etc.) in $\Psi$ (Eq. 1). Figure 3 shows the variations of $K_{p}$ distributions with respect to $B_{\text {tot }}$ within different $V_{s w}$ bins. The direct correlation between the average $K_{p}$ and $B_{\text {tot }}$, shown in Fig. 3 is similar to that in panel (c) of Fig. 1, but the individual $K_{p}$ distribution in each $V_{s w}$ bin is much better defined with less uncertainty because of less variability in the underlying conditions. 


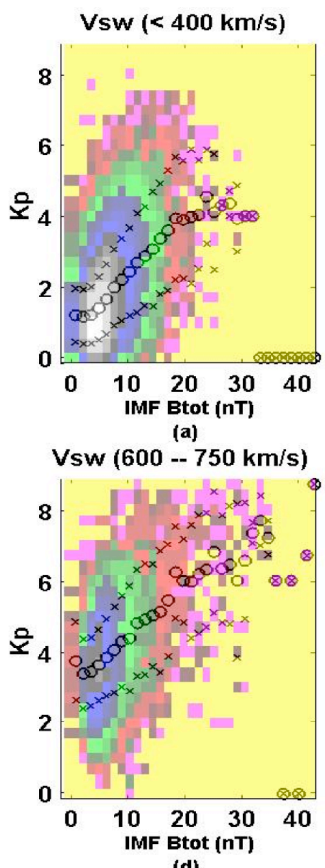

(d)

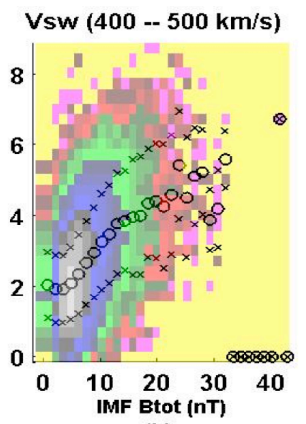

(b)

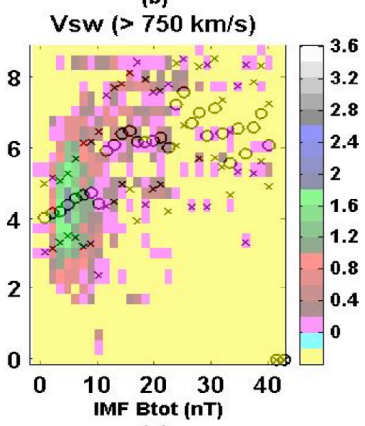

(e)

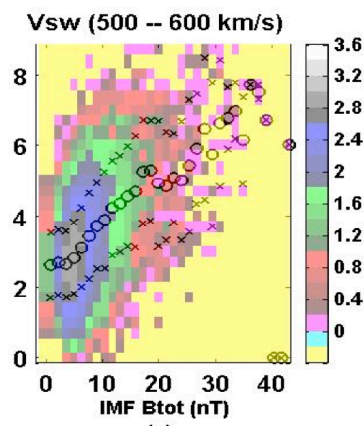

(c)

Fig. 3. (a) to (e) show the reduced histogram of $K_{p}$ index as a function of IMF magnitude $B_{\text {tot }}$ after localizing the solar wind speed $V_{s w}$ : (a) $V_{s w}<400 \mathrm{~km} \mathrm{~s}^{-1}$; (b) $400 \mathrm{~km} \mathrm{~s}^{-1} \leq V_{s w}<500 \mathrm{~km} \mathrm{~s}^{-1}$; (c) $500 \mathrm{~km} \mathrm{~s}^{-1} \leq V_{s w}<600 \mathrm{~km} \mathrm{~s}^{-1}$;

(d) $600 \mathrm{~km} \mathrm{~s}^{-1} \leq V_{s w}<750 \mathrm{~km} \mathrm{~s}^{-1}$; and (e) $750 \mathrm{~km} \mathrm{~s}^{-1} \leq V_{s w}$. The different panels, constructed similarly to those in Fig. 1, clearly reveal the inherent variation of (average) $K_{p}$ with both $B_{\text {tot }}$ and $V_{s w}$; whereas Fig. 1 shows only the overall $K_{p}$ variability with a single parameter. Compared to Fig. $1 \mathrm{~b}$ and c, the localized $K_{p}$ distributions in different $V_{s w}$ bins are more compact, yielding more accurate $K_{p}$ values to characterize magnetospheric states.

The distributions, as shown in Fig. 3, will allow us to compute the occurrence probability of a magnetospheric state specified by that parameter bin combination. Although a complete characterization of all magnetospheric state occurrences is beyond the scope of the present paper, we can see in the example of Fig. 3 that except for a very small number of cases (15 out of all the hourly intervals in 19702003), there is virtually no $K_{p}$ observed when $B_{\text {tot }}>30$ nT and $V_{s w}<500 \mathrm{~km} \mathrm{~s}^{-1}$. Conversely, there is a tendency for average $K_{p}>4$ when $B_{\text {tot }}>3 \mathrm{nT}$ and $V_{s w}>750 \mathrm{~km} \mathrm{~s}^{-1}$. It will be of interest, and quite straightforwardly so, to find out the conditions that led to the extreme event occurrences using the analysis technique described herein.

\subsection{Determination of $\boldsymbol{\tau}$}

So far, we have only assumed $\tau=0$ in Eq. (1). Although there may not be a significant arrival time difference between different solar wind and IMF drivers, say, at the front of the magnetosphere, different magnetospheric responses, such as the development and decay of ring currents and auroral processes, will have different response times. The time histories of different input may also have pre-conditioning effects on the magnetosphere, but here we wish to first determine the appropriate nominal time lags $\boldsymbol{\tau}=\left[\tau_{1}, \tau_{2}, \tau_{3}\right]$ of the multi- geomagnetic activities (see Eq. 1), whose responses would have implicitly taken time history into account. Then each of the geomagnetic indices in Eq. (1) can be replaced by its appropriately time-delayed values, such that Eq. (1) becomes (again ignoring $F 10.7$ for the time being)

$$
\begin{aligned}
& \boldsymbol{\Psi}(t)=\left[V_{s w}(t), B_{\mathrm{tot}}(t), P_{s w}(t), B_{z}(t) ; K_{P}\left(t+\tau_{1}\right), D_{s t}\left(t+\tau_{2}\right),\right. \\
& \left.A E\left(t+\tau_{3}\right), A L\left(t+\tau_{3}\right)\right] .
\end{aligned}
$$

It is immediately clear that the explicit time dependence $(t)$ in Eq. (2) can be dropped without loss of generality. A magnetospheric state $\boldsymbol{\Psi}$ at any solar wind/IMF arrival time $t$ is then simply specified by the magnetospheric driver and response parameters at appropriate time lags.

As described above, the localization process minimizes the systematic variations of parameters so that only a random error remains in a given localized parameter bin. The optimal time lag $\left(\tau_{1}, \tau_{2}, \tau_{3}\right.$ in Eq. 2$)$ of a given geomagnetic response can therefore be determined empirically by fixing the time lag at which the given index has a narrowest distribution, i.e. with a minimum standard deviation $\sigma$

$\sigma=\sqrt{\frac{\sum_{i=1}^{n}\left(G_{i}-\langle G\rangle\right)^{2}}{n}}$, 


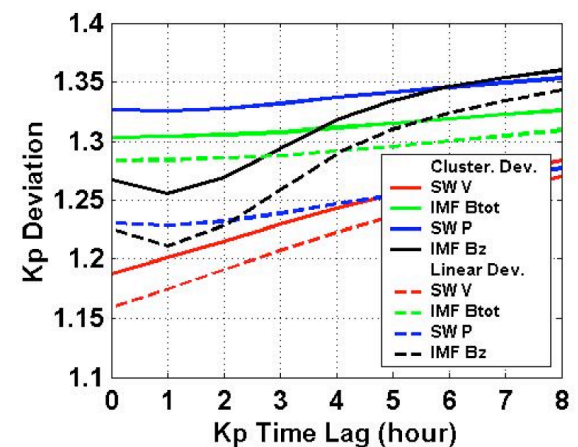

(a)

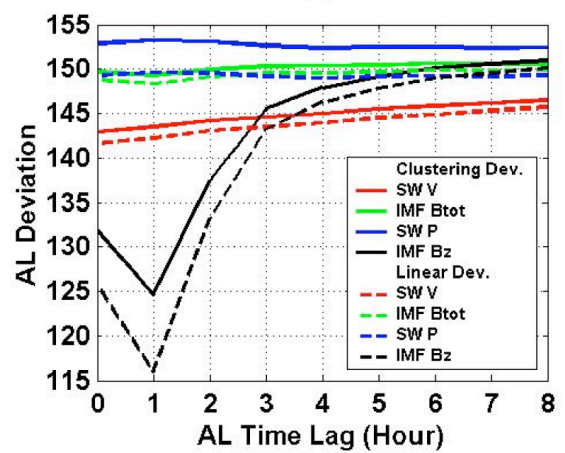

(c)

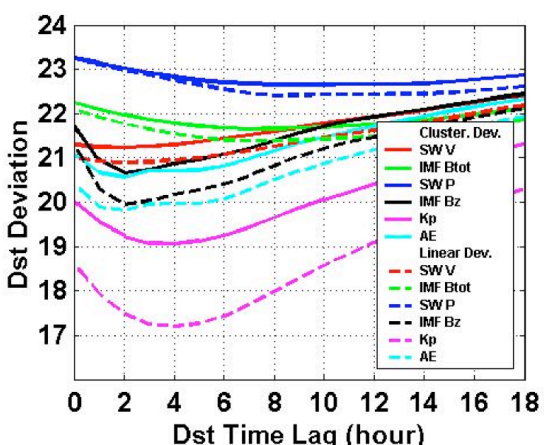

(b)

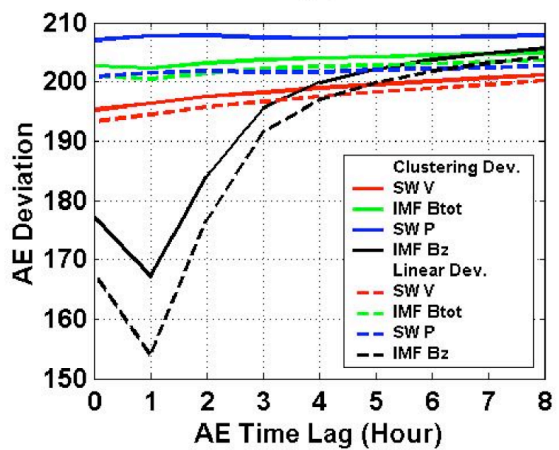

(d)

Fig. 4. Panels (a-d) show the total clustering deviations $\left\langle\sigma>_{c}\right.$ (solid color lines) and piecewise-linear deviations $\langle\sigma\rangle_{p}$ (dashed color lines) as a function of time lag of $K_{p}, D_{s t}, A E$, and $A L$, respectively. The time lag at which a given response index has the minimum deviation indicates the natural response time of that index to the corresponding input. Panel (b) indicates that $D_{s t}$ also responses to 3-h $K_{p}$ with a 2-5 h delay.

where $G_{i}$ are the measured values of the geomagnetic index ( $K_{p}, D_{s t}$, hourly $A E$, or hourly $A L$ ) and $n$ is the number of values in a given bin. The average value $\langle G\rangle$ can be obtained by computing the arithmetic mean of $G_{i}$ (as in Figs. 1 and 3 ) or finding the linear fit through the distribution within the localized-parameter bin. By simply averaging all the standard deviations in all bins (Duda et al., 2000),

$$
\langle\sigma\rangle=\sqrt{\sum_{j=1}^{m} \sigma_{j}^{2} n_{j} / \sum_{j=1}^{m} n_{j}}
$$

where $m$ is the number of bins of a given input parameter, we have obtained the total clustering standard deviation $\langle\sigma\rangle_{c}$ (if $\langle G\rangle$ is given by the arithmetic mean) and the total piecewise-linear standard deviation $\langle\sigma\rangle_{p}$ (if $\langle G\rangle$ is given by the piecewise linear fits).

Computed by using the magnetospheric state parameter data (see Eq. 1) taken in 1970-2003, Fig. 4a-d shows the $<\sigma>_{c}$ and $\left\langle\sigma>_{p}\right.$ as functions of lag time at which the $K_{p}$, $D_{s t}, A E$, and $A L$ time series are delayed from and correlated with various other state parameters. It is apparent that $\langle\sigma\rangle_{c}$ is generally larger than $\langle\sigma\rangle_{p}$ in all cases. The zero-lag case in Fig. 4a gives the net deviation of the $K_{p}$ distributions in $V_{s w}, B_{\text {tot }}, P_{s w}$, and $B_{z}$, as shown in Fig. 1 . It shows that $K_{p}$ values (though at 3 -h resolution) are best organized (with a most compact distribution) by the hourly-averaged $V_{s w}$ at zero lag. With respect to IMF $B_{z}$, however, the $K_{p}$ distribution in Fig. 1f would have been more compact if $K_{p}$ were delayed from IMF $B_{z}$ by about an hour, although there may actually be a range of valid lag of 0 to $2 \mathrm{~h}$. Thus, a 2-h history of IMF $B_{z}$ may be suitable for $K_{p}$ specification. Since $K_{p}$ has different effective lag times from $V_{s w}$ and $B_{z}$, it is therefore not likely to be dependent on the interplanetary electric field $E_{y, i, n}=V_{s w, i} B_{z, n}$.

On the other hand, Fig. 4a shows significant smaller variations of $\left\langle\sigma>_{p}\right.$ with $K_{p}$ lags for $B_{\text {tot }}$ and $P_{s w}$ as drivers. Both $\langle\sigma\rangle_{p}$ and $\langle\sigma\rangle_{c}$ start from their minima at zero lag (like the case of $V_{s w}$ ) and remain relatively constant until after a lag of about $2 \mathrm{~h}$, and then start to increase, indicating that these magnetohydrodynamic (MHD) drivers can elicit a fairly quick magnetospheric response that may last up to a few hours. Therefore, Fig. 4a gives the basic lags of $K_{p}$ from the solar wind and IMF input, and the nominal intervals of driver histories needed to specify the $K_{p}$ response. The most geo-effective drivers, i.e. to which $K_{p}$ is most sensitive, are clearly $V_{s w}$ and IMF $B_{z}$, which show well-defined minima and the largest changes in $\langle\sigma\rangle$ with lag.

Figure $4 \mathrm{~b}$ shows a more complicated time delay and inputdriver history dependence of $D_{s t}$. The hourly $D_{s t}$ index 


\begin{tabular}{|c|c|c|c|c|c|c|}
\hline Drivers & $\overline{V_{s w}}$ & $B_{t o t}$ & $I M F B_{z}$ & $P_{s w}$ & $K_{p}$ & $A E$ \\
\hline$K_{p}$ & 0 & 0 & $0-2$ & $0-3$ & - & - \\
\hline$D_{s t}$ & $0-4$ & $4-8$ & $2-3$ & $2-14$ & $2-5$ & $1-2$ \\
\hline$A E$ & 0 & $0-2$ & 1 & $0-8$ & - & - \\
\hline$I M F B_{z}$ & \multicolumn{3}{|c|}{$\begin{array}{c}V_{s w}, B_{t o t}, P_{s w} \\
K_{p}, A E\end{array}$} & \multicolumn{3}{|r|}{$D_{s t}$} \\
\hline$t-3$ & & -2 & & $t-1$ & & \\
\hline
\end{tabular}

Fig. 5. Relative time shifts (h) between driver and response parameters. $D_{s t}$ actually responses to $K_{p}$ and $A E$ (thus is sensitive to existing magnetospheric conditions), as well as to solar wind and IMF.

responds primarily to $K_{p}$ with about a 2-5-h delay, to $A E$ with a 1-2-h delay and to IMF $B_{z}$ with a 2-3-h nominal delay. A 4-h time history of $V_{s w}$ may also be important. On the other hand, $B_{\mathrm{tot}}$ and $P_{s w}$ seem to have little or very weak direct influence on the $D_{s t}$ index, as it has a rather long response time of $8-9 \mathrm{~h}$. The relatively weak $D_{s t}$ response to these MHD parameters suggests that $D_{s t}$ perturbations are not the direct result of pressure-driven MHD processes. The more sensitive $D_{s t}$ response to $K_{p}$, however, is understandable because the decay of $D_{s t}$ involves only processes within the magnetosphere (e.g. ring current decay). These processes are not driven directly by the solar wind and IMF, but are more dependent on the prevailing magnetospheric and ionospheric conditions, which may be globally characterized by $K_{p}$. Figure $4 \mathrm{~b}$ clearly shows the importance of accounting for the effects of other geomagnetic responses, in addition to the driver parameters, when predicting $D_{s t}$.

Figure $4 \mathrm{c}$ and $\mathrm{d}$ shows the time delay and input-driver history dependence of $A L$ and $A E$, respectively. Both plots indicate that the auroral indices respond primarily and most sensitively to the IMF $B_{z}$ input with a lag of an hour. Their responses to other driver parameters are rather weak, except perhaps for $V_{s w}$, to which the auroral indices could have moderate responses within the hour of input (zero lag). The similarity between these two figures is expected because of the close relation between the $A E$ and $A L$ indices, and we can drop $A L$ from Eqs. (1) and (2).

Figure $4 a-d$ confirms that a given magnetospheric driver can give rise to multiple magnetospheric responses via processes (at different locations) having different time (and spatial) scales. As we discussed earlier about Fig. 4a, more geoeffective drivers tend to lead to well-defined minima and significant changes in the $<\sigma>-$ lag curves. For those drivers, the magnetospheric response times are conveniently given by the times of the curve minimum. Less sensitive input drivers can still be effective if their actions are sustained for a sufficiently long period of time, in which case the integrated driver time history may be more important.
Figure 5 summarizes the nominal ranges of relative time shifts (h) between the different magnetospheric drivers and response parameters. The finite lag time ranges suggest the intervals of effective time history of different input parameters. In the present study, however, we focus only on choosing a set of specific delay times that are consistent with the delay time ranges in order to obtain a nominal set of relative timing relationships among each of the state parameters. The results are shown at the bottom of Fig. 5. For the particular set of chosen time delays in Fig. $5, D_{s t}$ has the longest response time to solar wind and IMF input. It also responds to past $K_{p}$ and $A E$ conditions that may govern ring current buildup and decay processes. In addition, since Fig. $4 \mathrm{~b}$ indicates that $D_{s t}$ has only a relatively weak IMF- $B_{\text {tot }}$ dependence with a $4-8 \mathrm{~h}$, we have simply chosen a $2-\mathrm{hr}$ delay between $D_{s t}$ and IMF- $B_{\text {tot }}$, so that a consistent set of response times to IMF $B_{\text {tot }}$ can be chosen for $K_{p}$ and $A E$, as well.

In order to see how well the set of time delays given in Fig. 5 can help reduce the effects of systematic variability, as discussed in Sect. 4.1, we show in Fig. 6a-c the continuous decreases of $\langle\sigma\rangle_{p}$ and $\langle\sigma\rangle_{c}$ of the $K_{p}, A E$, and $D_{s t}$ indices, as the multi-responses are appropriately delayed and correlated with successively localized parameters. We should emphasize, however, that data bins having unphysical combinations of parameter values (such as low $V_{s w}$ and high $\left.K_{P}\right)$ are of little interest. Extreme events, like great storms with $D_{s t}<300 \mathrm{nT}$, are rare; and they need to be treated separately.

Using the optimal delays in Fig. 5 and ignoring the timeintegrated effects for the time being, Eq. (2) then becomes (again ignoring $F 10.7$ and $A L$ as before)

$$
\begin{aligned}
& \Psi(t)=\left[V_{s w}(t-2 \mathrm{~h}), B_{\mathrm{tot}}(t-2 \mathrm{~h}), P_{s w}(t-2 \mathrm{~h}), B_{z}(t-3 \mathrm{~h})\right. \\
& \left.K_{P}(t-2 \mathrm{~h}), D_{s t}(t), A E(t-2 \mathrm{~h})\right] .
\end{aligned}
$$

The vector $\boldsymbol{\Psi}(t)$ in Eq. (5) specifies the instantaneous state of the magnetosphere, which can be viewed as a snapshot of the global magnetospheric configuration (see discussion of Eq. 2), though it does not tell us how the magnetospheric state evolves. Temporal changes in $\boldsymbol{\Psi}(t)$ are manifested only in the collective progression of individual state parameters, as in Fig. 7a. We should note that $\boldsymbol{\Psi}(t)$ depends on $V_{s w}, B_{\text {tot }}$, $P_{s w}, B_{z}, K_{p}$ and $A E$ at hours previous to that of $D_{s t}$; so both "historical" and current information are used to specify the magnetospheric state at $D_{s t}$ time. Nevertheless, incorporation of parameter histories can lead to refinement of magnetospheric state specification.

\section{Validating multi-response specifications}

The notion of a magnetospheric state is meaningful only if a given combination of magnetospheric-state parameter ranges in Eq. (5) always represents the same magnetospheric configuration, and it is repeatable. Different parameter range combinations can therefore serve as identifiers (or labels) of 

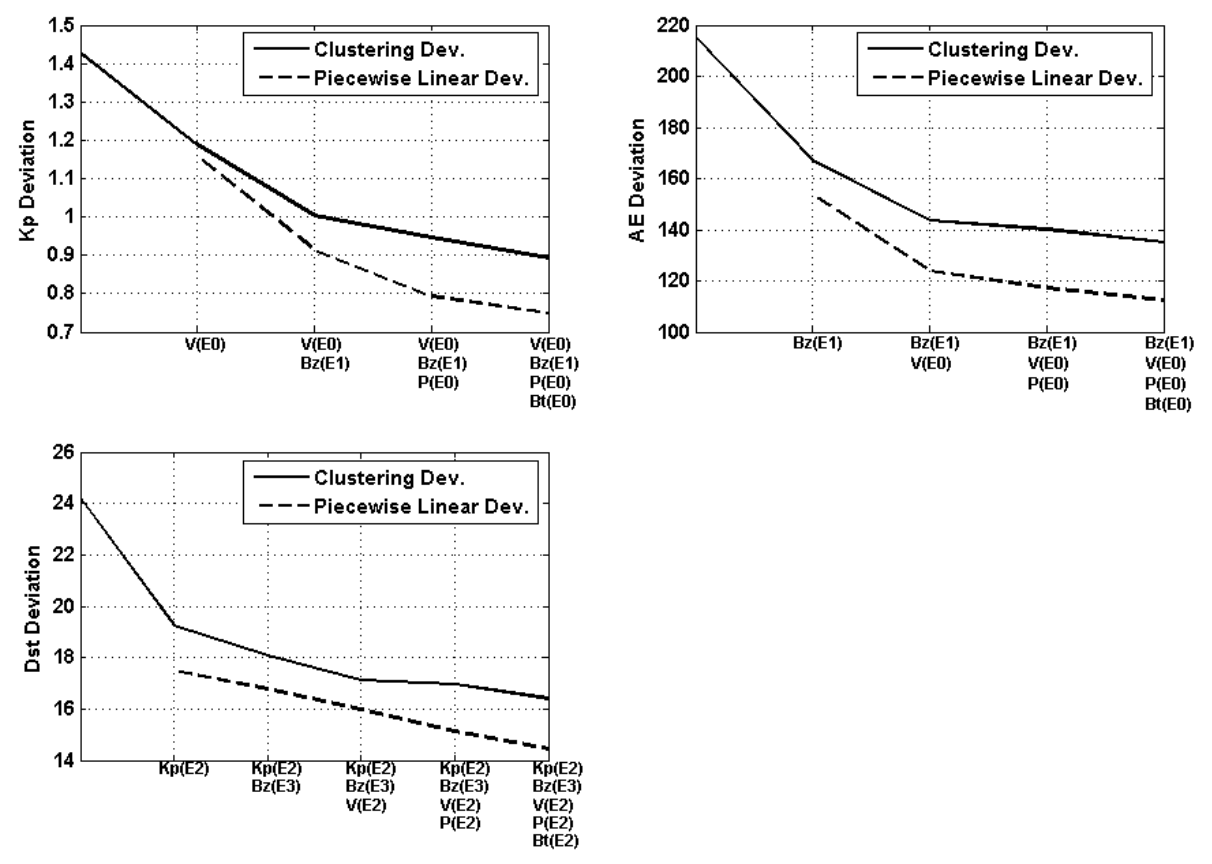

Fig. 6. (a) to (c) show the resultant decreases in $\left\langle\sigma>_{p}\right.$ and $\left\langle\sigma>_{c}\right.$ of $K_{p}, A E$, and $D_{s t}$, respectively, when the delays of $K_{p}, A E$, and $D_{s t}$ responses are incorporated in the successive localization process: (a) deviation of $K_{p}$ distribution when $V_{s w}(\mathrm{E} 0)$, IMF $B_{z}(\mathrm{E} 1), P_{s w}(\mathrm{E} 0)$, and IMF $B_{\text {tot }}(\mathrm{E} 0)$ are successively localized; (b) deviation of $A E$ distribution when IMF $B_{z}(\mathrm{E} 1), V_{s w}(\mathrm{E} 0), P_{s w}(\mathrm{E} 0)$, and IMF $B_{\mathrm{tot}}(\mathrm{E} 0)$ are successively localized; (c) deviation of $D_{s t}$ distribution when $K_{p}(\mathrm{E} 2), V_{s}(\mathrm{E} 2), \mathrm{IMF} B_{z}(\mathrm{E} 3), P_{s w}(\mathrm{E} 2)$, and IMF $B_{\text {tot }}(\mathrm{E} 2)$ are successively localized. Here E0 and E1 indicate 0 and $1 \mathrm{~h}$, respectively, earlier than the geomagnetic index observations. The localization processes clearly reduce the deviations significantly.

different magnetospheric states, analogous to using spectroscopic terms, to identify different energy states or electronic configurations of an atom. Since the multi-response parameters $\left(K_{p}, D_{s t}\right.$, and $\left.A E\right)$ must somehow be "determined" by the input drivers, the validity of the magnetospheric state prescriptions can be verified by checking the correspondence between the magnetospheric driver and response parameters.

To test this idea, we have used the long-term solar wind and IMF data available from the NASA Space Science Data Center (NSSDC) to determine the various possible driver state bins $D_{i, j, k, n}=\left[V_{s w, i}(t-2 \mathrm{~h}), B_{\mathrm{tot}, \mathrm{j}}(t-2 \mathrm{~h})\right.$, $\left.P_{s w, k}(t-2 \mathrm{~h}), B_{z, n}(t-3 \mathrm{~h})\right]$ as described above. For each $D_{i, j, k, n}$ then, we can determine the corresponding response state by computing the set of expectation values $\left\langle R_{i, j, k, n}\right\rangle$ of the response parameters $R=\left[K_{P}(t-2 \mathrm{~h})\right.$, $\left.D_{s t}(t), A E(t-2 \mathrm{~h})\right]$. The expectation values $\left\langle R_{i, j, k, n}\right\rangle$ are computed by taking either the arithmetic mean or the piecewise-linear fit of each of the response parameters sampled within each $D_{i, j, k, n}$, depending on whether the number of samples $n$ is less than or greater than $80(\sqrt{n} / n \sim 0.11)$, respectively. For $D_{s t}$, however, results in Fig. $4 \mathrm{~b}$ suggest that $\left.<D_{s t}\right\rangle$ should be calculated only after $K_{p}$ is also localized. With these definitions, we then have the following complete specifications of magnetospheric states:

$\boldsymbol{\Psi}=\left[D_{i, j, k, n},<R_{i, j, k, n}>\right]$ with $i, j, k=1,2,3,4,5$ and $n=1,2,3,4,5,6$.

Using the historical solar wind, IMF and geomagnetic indices data (1970-2000), we have statistically determined the different $\left.<R_{i, j, k, n}\right\rangle$ that correspond to all possible driver states $D_{i j k n}$, assuming that the magnetosphere had experienced all possible states with sufficient recurrences of each state (except those associated with rare events) over the historical data period. Applying the established correspondence between $<R_{i, j, k, n}>$ and $D_{i, j, k, n}$, we can then prescribe the multi-geomagnetic responses to the observed $D_{i, j, k, n}$ at any time.

\subsection{A magnetic cloud event}

Figure 7a shows an example of applying the results from Eq. (6) to specify simultaneously the $K_{p}, D_{s t}$, and $A E$ responses (with different time shifts as shown in Fig. 5) to the observed solar wind and IMF input during a magnetic cloud event which occurred on 18-19 October 1995 (Fenrich and Luhmann, 1998). In this example, the correlation coefficients between the specified (red curves) and observed (black curves) values of $K_{p}, D_{s t}$, and $A E$ indices are $0.83,0.92$, and 0.90 , respectively. As we can see, the magnetospheric state prescriptions capture very well simultaneously the multiple geomagnetic responses to the variable solar wind and IMF input. The blue (solid and dashed) vertical lines indicate 

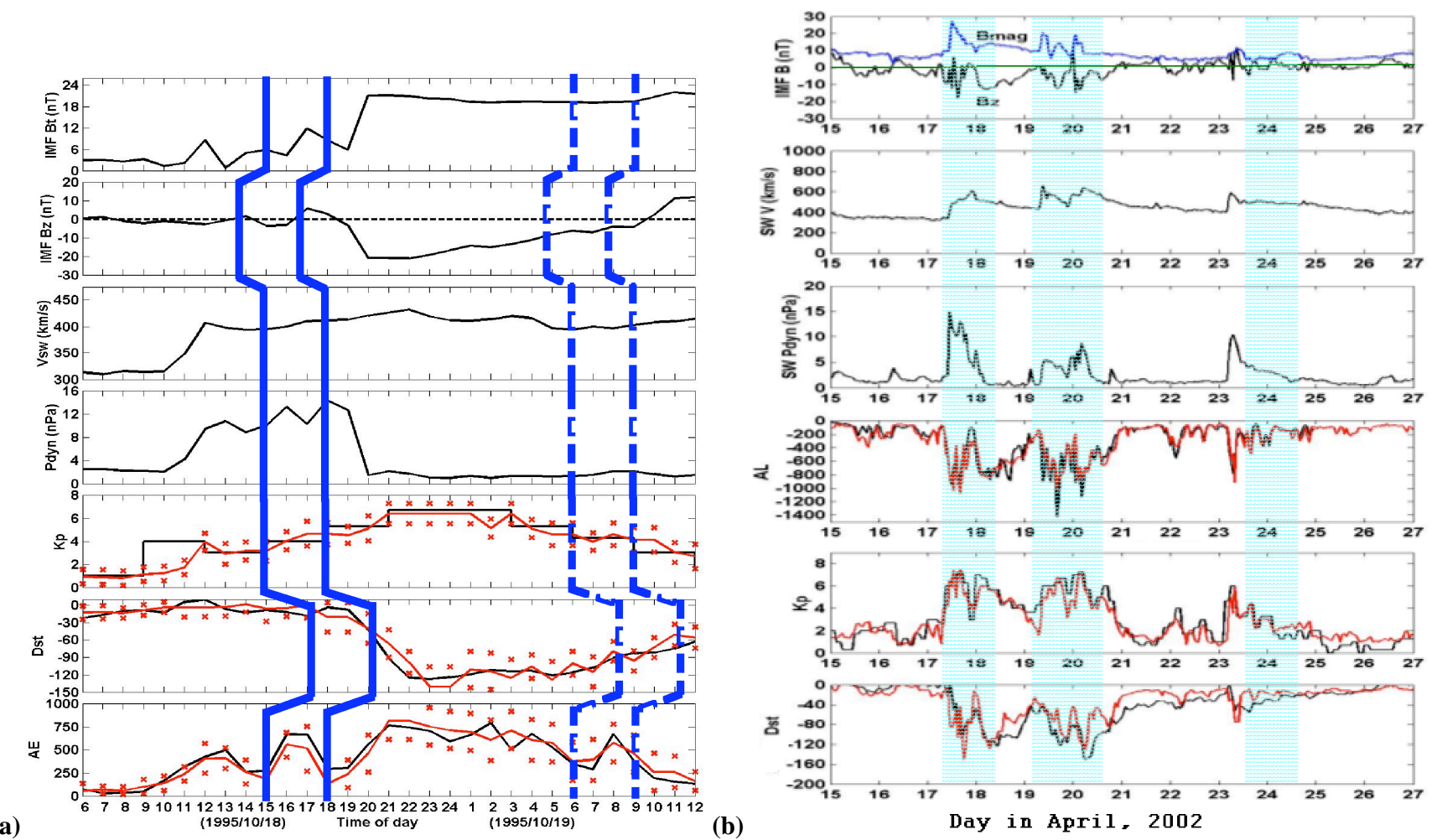

Fig. 7. (a) Evolution of magnetospheric states, $\Psi(t)$, over a magnetic cloud interval on 18-19 October 1995 (Fenrich and Luhmann, 1998) is manifested by the continuously changing magnetospheric state parameters. With a fixed set of time shifts, as marked by the blue (solid and broken) lines, the temporal progressions of the time-series records of the different magnetospheric state parameters simply mark the temporal changes in the magnetospheric states. The red curves show average prescribed geomagnetic responses in remarkable agreement with observations (black curves). The red crosses give a one standard deviation error estimate. (b) Illustration of successful simultaneous specification of $A L, K_{p}$, and $D_{s t}$ by magnetospheric state prescriptions (with mean square-root error of 131.2, 0.94, and 21.3, respectively) over an interval of multiple storms with varying magnitudes observed on 15-27 April 2002. The prescribed geomagnetic indices (red curves) and their observed values (black curves) have correlation coefficients of $0.89,0.90$, and 0.83 , respectively.

the correspondence between the time-shifted magnetospheric drivers and responses at four different times. They show that the prescriptions given by Eq. (6) are able to specify correctly the similar $K_{p}$ and $A E$, but quite different $D_{s t}$ responses between the two sample intervals bounded by the solid and dashed blue lines, owing to the two intervals having significantly different driver input.

\subsection{A period of multiple storms}

Figure $7 \mathrm{~b}$ shows another example interval of 15-27 April 2002, when multiple storms with varying magnitudes were observed over a 12-day period. Again, the simultaneously specified $A L, K_{p}$ and $D_{s t}$ indices show good agreement with their corresponding observed values, with correlation $>0.83$. The three periods with varying degrees of disturbances (shaded blue) within the interval show that all three periods have very similar general driver conditions: enhanced $B_{\text {tot }}$, generally $B_{z}<0$, elevated $V_{s w}$, and sharp increases in $P_{s w}$. They also have very similar magnetospheric responses correspondingly, and thus support the notion of magnetospheric states prescribed by Eq. (6).

\subsection{The years of 2001 and 2002}

Magnetospheric state approach is correct only if it is applicable and valid for arbitrarily long time intervals. In addition to the two intervals of different lengths described above, we have investigated the performance of Eq. (6) over extended periods. Figures 8, 9, and 10 show the comparisons between the prescribed (red curves) and observed (black curves) long-term $\left\langle K_{p}\right\rangle$ and $\left\langle D_{s t}\right\rangle$ in 2002, and $<A E>$ in 2001, respectively. The agreements between all the prescribed $\left\langle R_{i, j, k, n}\right\rangle$ and their corresponding actual measurements are quite remarkable, with correlation coefficients $>0.75$ (see captions of Figs. 8-10).

In the next section, we compare in more detail the multiparameter specification technique investigated here and other geomagnetic response prediction models. 


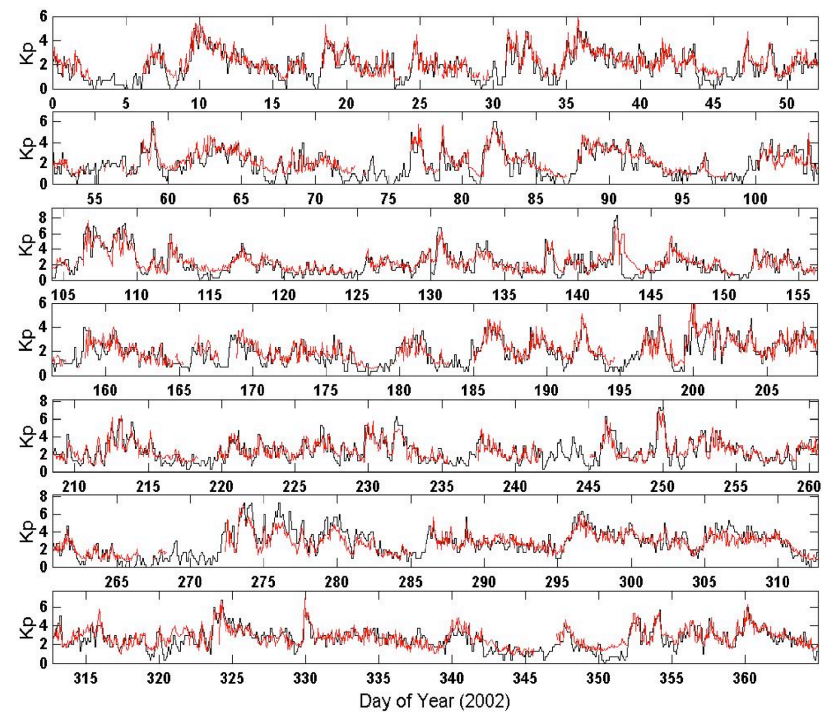

Fig. 8. The $K_{p}$ index values as a function of time in the year 2002 are specified by their corresponding driver states, in accordance with the magnetospheric state prescription of Eqs. (5) and (6). Magnetospheric states are specified by the driver and response parameter data obtained in 1970-2001, with $V_{s w}(\mathrm{E} 0), B_{z}(\mathrm{E} 1), P_{s w}(\mathrm{E} 0)$, and $B_{\text {tot }}(\mathrm{E} 0)$ having been localized. The estimation (RMS) error is \pm 0.795 [in $K_{p}$ unit]; consistent with the global deviation shown in Fig. 6a and correlation factor with the actual data is 0.801 . Black line is the actual observed $K_{p}$ index and red line is the estimated $K_{p}$ index. The gaps in the red line indicate periods of no solar wind data.

\section{Comparisons with existing geomagnetic response models}

Neural network models have been developed in the past for separate prediction of $K_{p}, D_{s t}$ and $A E$ (e.g. Takalo and Timonen, 1997; Gavrishchaka and Ganguli, 2001; Boberg et al., 2000; Lundstedt et al., 2002). The root-mean squared (RMS) error of $K_{p}$ prediction in the Boberg et al. model is $\sim 0.8$. With the magnetospheric state specification technique, we have achieved comparable accuracy (see Fig. 8 caption). Unlike the neural-network models for $K_{p}$ prediction, however, the magnetospheric state prescriptions also allow the simultaneous specifications of $D_{s t}$ and $A E$ responses that have different variability time scales. Since different geomagnetic responses are not completely independent from one another because they are all mediated by the same magnetospheric magnetic field, it is important to take into account their interdependences when we consider the behavior of a given response.

An important distinction between the magnetospheric state specification and the familiar linear-filter techniques is that magnetospheric prescriptions (Eq. 6) allow all $<R_{i, j, k, n}>$ to be specified simultaneously over long period ( days) without any special processing or modeling of the input-driver data. The prescriptions given by Eq. (6) spec-

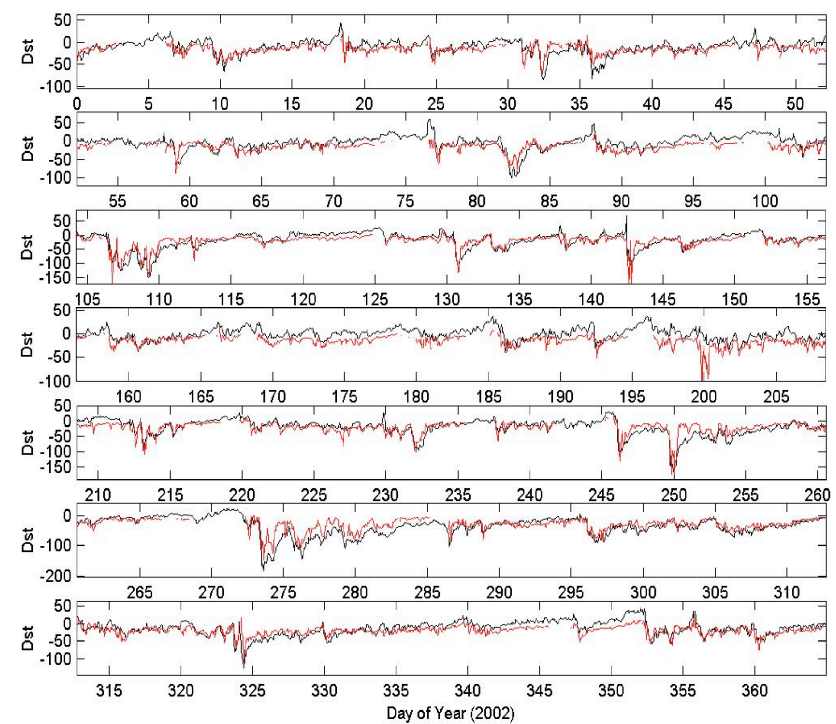

Fig. 9. The $D_{s t}$ index values as a function of time in the year 2002 are specified by their corresponding driver states, in accordance with the magnetospheric state prescription of Eqs. (5) and (6). Magnetospheric states are specified by the driver and response parameter data obtained in 1970-2001, with $K_{p}(\mathrm{E} 2), V_{s w}(\mathrm{E} 2), B_{z}(\mathrm{E} 3)$, $P_{s w}(\mathrm{E} 2)$, and $B_{\mathrm{tot}}(\mathrm{E} 2)$ having been localized. The estimation error is $\pm 16.7 \mathrm{nT}$ (consistent with Fig. 6c) and correlation factor with the actual data is 0.757 . The black line is the actual observed $D_{s t}$ index and red line is the estimated $D_{s t}$ index. The gaps in the red line indicate periods of no solar wind data.

ify the global multi-responses of the magnetosphere, instead of just a single output parameter. The residual differences between $\left\langle R_{i, j, k, n}\right\rangle$ and their measured values, particularly in the case of $D_{s t}$, are perhaps due to the so-far incomplete accounting of the time histories of the magnetospheric state parameters.

There has also been nonlinear models developed to predict minute-resolution $A L$ and $A E$ indices during isolated substorms (e.g. Vassilidias et al., 1995), but success is lacking in predicting the long-term evolution of the electrojet indices due to multiple upstream input variability. From the present work, we can see that although substorm activities can occur on minute-to-hour scales, the overall hourly highlatitude ionospheric current systems activities can be specified by the hourly-averaged solar wind and IMF conditions (see Eq. 6), although variations of ionospheric conditions as parameterized by $F 10.7$ radio fluxes have so far been neglected. Figure 5 shows that both $K_{p}$ and $A E$ indices are direct consequences of magnetospheric input drivers. The different response times of the indices to different (hydrodynamic or magnetic) driver parameters imply that different geo-effective processes contribute to different geomagnetic activities. The incorporation of multiple responses in Eq. (6) seems to be key to the apparent success of magnetospheric state prescriptions. 


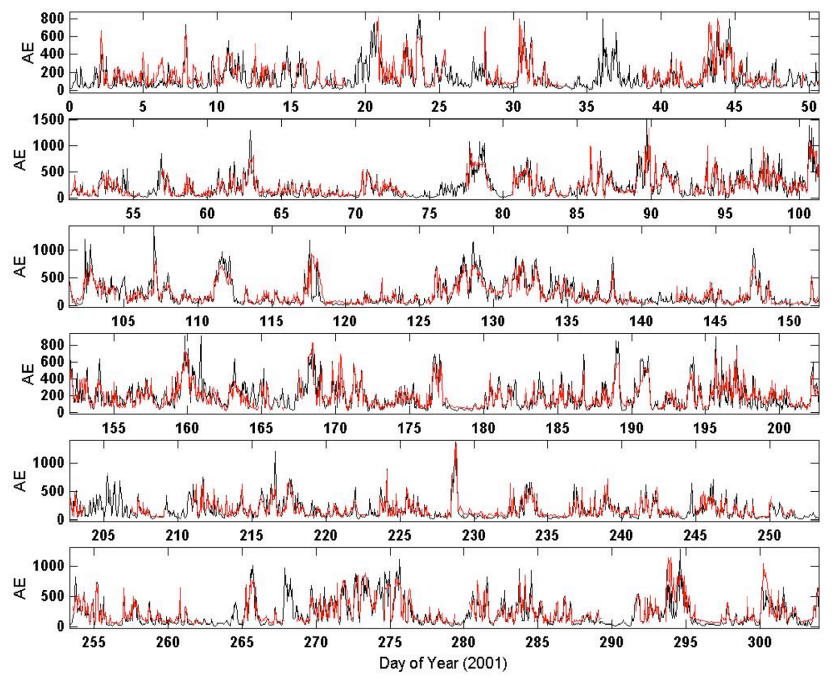

Fig. 10. The $A E$ index values as a function of time in 10 months of 2001 are specified by their corresponding driver states, in accordance with the magnetospheric state prescription of Eqs. (5) and (6). Magnetospheric states are specified by the driver and response parameter data obtained in 1970-2000, with $V_{s w}(\mathrm{E} 0)$, $B_{z}(\mathrm{E} 1), P_{s w}(\mathrm{E} 0)$, and $B_{\text {tot }}(\mathrm{E} 0)$ having been localized. The estimation error is $\pm 111.8 \mathrm{nT}$ (consistent with Fig. $6 \mathrm{~b}$ ) and correlation factor with the actual data is 0.826 . The black line is the actual observed $A E$ index and red line is the estimated $A E$ index. The gaps in the red line indicate periods of no solar wind data.

Because of the strong contextual relationship between geomagnetic storm activities and the $D_{s t}$ index, there has been particular interest in $D_{s t}$ predictions (e.g. Burton et al., 1975; Klimas et al., 1998; O'Brien and McPherron, 2000; Temerin and Li, 2002; Lundstedt et al., 2002). Models in the past have invariably endeavored to provide the time evolution of $D_{s t}$ by modeling heuristically the $D_{s t}$ growth and decay rates as functions of solar wind and IMF input. One of the most successful models so far is perhaps the Temerin and Li (2002) model with $\sim 90 \%$ prediction efficiency. A common feature of most of these models, however, is the requirement of past fiduciary $D_{s t}$ values. The neural network model by Lundstedt et al. (2002) does use multiple driver parameters $\left(V_{s w}\right.$, $B_{z}$ and $\left.n_{s w}\right)$ to predict $D_{s t}$ without having to use any past fiduciary values, but it neglects to account for the effects of other magnetospheric responses that must also affect $D_{s t}$, as geomagnetic activities are not independent of one another.

The $D_{s t}$ index (sometimes corrected for solar wind pressure effects and magnetospause current contributions) is largely a measure of the ring current activities that has long decay times ( $\sim$ few days) due to collisional and chargeexchange loss processes that are dependent on prevailing magnetospheric conditions. Figure 5, in fact, shows that $D_{s t}$ does depend on earlier $K_{p}$ and $A E$ indices with different response times, although a consistent two-hour delay has been chosen in this study for convenience. The incorpora- tion of the different response times of the multi-geomagnetic responses in the magnetospheric state specifications is analogous to performing multivariate analysis of a nonlinear system. Consequently, unlike $D_{s t}$ prediction models in the past, our specification technique requires no prior $D_{s t}$ information and does take into account the underlying geomagnetic responses in order to prescribe the $D_{s t}$ component of a particular magnetospheric state vector (Eq. 5). As shown in Fig. 9, the primary temporal variations of $D_{s t}$ are reasonably captured by the rather simple, but straightforward implementation of magnetospheric state prescriptions (Eq. 6).

\section{Summary and discussion}

We have investigated the simultaneous specification of the multi-geomagnetic responses of the magnetosphere as a result of varying solar wind and IMF input. We have found that there exists a well-defined set of relative time differences among the various magnetospheric-state parameters, as shown in Fig. 5. These time differences are the different response times of the parameters relative to one another (see Figs. 1-4). Figure 6 shows that the response parameter distributions become more compact when their finite response times are properly accounted for and the systematic variations of the driver (independent) parameters are effectively minimized by successive localization.

Since the time-shifted magnetospheric driver and response parameters, according to Fig. 5, are correlated, their variation must also correspond to one another such that their time progressions should indicate the evolution of the state of the magnetosphere, as illustrated in Fig. 7a. Using a long interval of magnetospheric state parameter data (1970-2000) and following Eq. (5), we have obtained statistically a "look-up table" for the prescriptions of different magnetospheric states at an hourly resolution given by Eq. (6). The magnetospheric state look-up table is useful for performing cluster analysis, with which one can determine the conditions for the occurrences of different types of events of interest (e.g. storms or substorms), and forecast space weather events.

To test the validity of magnetospheric state prescriptions, we have applied the results from Eq. (6) to prescribe simultaneously the multi-geomagnetic responses $<R_{i, j, k, n}>$ that should correspond to the driver states $D_{, i, j, k, n}$ sequence observed in different data intervals. The results are shown in Figs. 7-10. Since the $D_{s t}$ index has the longest lag time of about $3 \mathrm{~h}$ (from IMF $B_{z}$ ), we can thus predict its responses with at least a few hour's lead time. Longer lead times are clearly possible if upstream driver conditions can be measured well in advance.

Unlike traditional neural-network or linear-prediction filter analyses, in which time-series data of solar wind and IMF input and a geomagnetic index output $\left(K_{p}, D_{s t}\right.$ or $\left.A E\right)$ have to be used to train and construct an input-output transfer function, the localization process used to minimize the 
systematic variation of parameters requires no special data processing or modeling. Once the relative time shifts (Fig. 5) between the state parameters are determined, their temporal relationships are then fixed for all times, as shown in Eq. (5) and Fig. 7a.

Although at the present time the magnetospheric state specification technique is less accurate than some specialized models, such as the Temerin and Li (2002) model, in predicting the $D_{s t}$ index, it is a more capable technique in that it can specify simultaneously multiple geomagnetic responses having different characteristic time scales. Such capability makes the technique very convenient and advantageous for space weather modeling and forecasting because the magnetospheric state paradigm can be applied similarly to develop different space environment specification models (Fung, 1996, 2004a; Fung et al., 2005) and greatly facilitate analyses (Fung et al., 2006).

Space physics event and statistical studies often require analyzing data taken under similar geophysical conditions, or in the same magnetospheric state. Since space physics data are time-ordered, selection and retrieval of multiple data intervals having similar geophysical conditions (characterized by multiple parameters) can be laborious. To facilitate data selection by magnetospheric conditions, we have developed the Magnetospheric State Query System (MSQS, http:// radbelts.gsfc.nasa.gov/RB_model_int/Psi_database.html) that can return a set of time intervals when the user-specified magnetospheric conditions are met (Fung, 2004b). Output from MSQS then allows the retrieval of all available data for the conditions of interest. The MSQS also allows queries for intervals of time-shifted parameters, such as those given in Fig. 5. It is ideal for testing and improving upon the magnetospepheric state prescriptions (Eq. 6).

Tsyganenko (2002) stated succinctly that in the case of data-based modeling, "one of the general goals (in magnetic field modeling) is to 'animate' the data-based models by enabling them to reproduce the continuous magnetospheric response to changing solar wind conditions." With the results of Eq. (6), it is possible to continuously specify the global magnetospheric state, and in turn, the magnetic field configuration (Tsyganenko, 2002) or the radiation belt (Fung, 1996; Fung et al., 1999, 2005). The magnetospheric state paradigm is equally applicable to other magnetospheric models, enabling a coherent understanding of the magnetosphere.

Tsyganenko (2002) pointed out also that early data-driven magnetic field models have been developed by dividing the entire data set into several subsets according to the $K_{p}$ activity levels. Thus different model coefficients were found for different $K_{p}$ bins. It was then found that when more than one model input parameter is used, the modeling technique then becomes unfeasible because there may not be enough observations to match the rapidly growing number of bins. This is not a problem for magnetospheric state specification per se, as there are no model coefficients involved. As we discussed in Fig. 7a, the same $K_{p}$ condition can arise from different magnetospheric states (having different corresponding solar wind and $D_{s t}$ conditions). So by using only data that are truly associated with similar conditions, the magnetospheric state technique actually helps reduce statistical errors, as illustrated in Fig. 6.

Finally, we note that there remain errors in the current magnetospheric state prescriptions. These errors may be attributable to the fact that only nominal time delays of the state parameters have been included for simplicity sake. Since the $F 10.7$ fluxes have been omitted in Eqs. (2) and (5), in order to simplify the present study, effects of changing ionospheric conditions controlled by the solar UV radiation have been neglected. Such effects may be important as they contribute to the chemical composition of the ionosphere, the plasmasphere, and thus the ring current decay processes. This may account for the relatively poor agreement between the prescribed and actual $D_{s t}$ during the decay phases of geomagnetic storms, as shown in Fig. 9. Other factors that have been neglected include: seasonal variations, magnetic dipole tilt effects, potential IMF $B_{y}$ and $B_{x}$ effects, and nonlinearity of the geomagnetic responses (e.g. $t=t\left(V_{s w}\right.$, IMF $\left.B_{z}\right)$; cf. Fig. 5). These considerations are beyond the scope of the present paper, but they will be addressed in future work.

Acknowledgements. We thank the World Data Center for Geomagnetism, Kyoto, Japan for providing the geomagnetic indices data and OMNIweb of the NASA National Space Science Data Center, Greenbelt, Maryland, USA, for providing the solar wind and interplanetary magnetic field data for this study. Work by Shao has been supported by the National Research Council Resident Research Associateship Program. This work is performed under NASA RTOP 784-50-51-02.

Topical Editor I. A. Daglis thanks two anonymous referees for their help in evaluating this paper.

\section{References}

Axford, W. I. and Hines, C. O.: A unifying theory of high-latitude geophysical phenomena and geomagnetic storms, Can. J. Phys., 39, 1433-1464, 1961.

Baker, D. N., McPherron, R. L., Cayton, T. E., and Klebesadel, R. W.: Linear prediction filter analysis of relativistic electron properties at 6.6 $R_{E}$, J. Geophys. Res., 95, 15 133-15 140, 1990.

Boberg, F., Wintoft, P., and Lundstedt, H.: Real time $K_{p}$ predictions from solar wind using neural networks, Phys. Chem. Earth, 25, 275-280, 2000.

Burlaga, L. F., Lepping, R. P., and Jones, J. A.: Global configuration of a magnetic cloud, in: Physics of magnetic flux ropes, American Geophysical Union, Washington, D.C., p. 373-377, 1990.

Burton, R. K., McPherron, R. L., and Russell, C. T.: An empirical relationship between interplanetary conditions and $D_{s t}$, J. Geophys. Res., 80, 4204-4214, 1975.

Clauer, C. R.: The technique of linear prediction filters applied to studies of solar wind-magnetosphere coupling, in: Solar Wind-Magnetosphere Coupling, Tokyo/Dordrecht, Terra Scientific Publishing Co./D. Reidel Publishing Co., p. 39-57, 1986. 
Duda, R. O., Hart, P. E., and Stork, D. G.: Pattern Classification, 2nd edition, Wiley-Interscience, 2000.

Dungey, J. W.: Interplanetary magnetic field and the auroral zones, Phys. Rev. Lett., 6, 47, 1961.

Fenrich, F. R. and Luhmann, J. G.: Geomagnetic response to magnetic clouds of different polarity, Geophys. Res. Lett., 25, 2999 3002, 1998.

Fung, S. F.: Recent development in the NASA trapped radiation models, in: Radiation belts Models and Standards, Geophys. Monogr., 97, AGU, Washington, D.C., 79-91, 1996.

Fung, S. F.: Survey of current situation in radiation belt modeling, Adv. Space Res., 34(6), 1441-1450, doi:10.1016/S02731177(03)00078-4, 2004a.

Fung, S. F.: Announcement of a "Magnetospheric-State Query System", American Geophysical Union SPA Section Newsletter, vol. XI, Issue 9, ftp://igpp.ucla.edu/scratch/aguspa/volume11_2004/ vol11no009, January 28, 2004b.

Fung, S. F., Bourgin, R. D., Cheung, C. Y., and Qian, S.: Development of a new-generation trapped radiation data base, in: Proceedings of the Space Radiation Environment Modeling Workshop, Farnborough, UK, 1-3 November 1999.

Fung, S. F., Bell, E. V., Tan, L. C., Candey, R. M., Golightly, M. J., Huston, S. L., King, J. H., and McGuire, R. E.: Development of a magnetospheric state-based trapped radiation database, Adv. Space Res., 36(10), 1984-1991, doi:10.1016/j.asr.2004.04.020, 2005.

Fung, S. F., Shao, X., and Tan, L. C.: Long-term variations of the electron slot region and global radiation belt structure, Geophys. Res. Lett., 33, L04105, doi:10.1029/2005GL024891, 2006.

Fung, S. F. and Tan, L. C.: Time correlation of low-altitude relativistic trapped electron fluxes with solar wind speeds, Geophys. Res. Lett., 25, 2361-2364, 1998.

Gavrishchaka, V. V. and Ganguli, S.: Optimization of the neuralnetwork geomagnetic model for forcasting larg-amplitude substorm events, J. Geophys. Res., 106, 6247-6257, 2001.

Gosling, J. T.: Coronal mass ejections and magnetic flux ropes in interplanetary space, in Physics of magnetic flux ropes, American Geophysical Union, Washington, D.C., p. 343-364, 1990.

Gussenhoven, M. S.: Low-altitude convection, precipitation, and current patterns in the baseline magnetosphere, Rev. Geophys., 26, 792-808, 1988.

Hoffman, R. A., Sugiura, M., Maynard, N. C., Candey, R. M., Craven, J. D., and Frank, L. A.: Electrodynamic patterns in the polar region during periods of extreme magnetic quiescence, $\mathrm{J}$. Geophys. Res., 93, 14 515-14 541, 1988.

Klimas, A. J., Vassiliadis, D., and Baker, D. N.: $D_{s t}$ index prediction using data-derived analogues of the magnetospheric dynamics, J. Geophys. Res., 103, 20 435-20 448, 1998.

Klimas, A. J., Baker, D. N., Roberts, D. A., Fairfield, D. H., and Buchner, J.: A nonlinear dynamical analogue model of geomagnetic activity, J. Geophys. Res., 97, 12 253-12 266, 1992.

Lundstedt, H., Gleisner, H., and Wintoft, P.: Operational forecasts of the geomagnetic $D_{s t}$ index, Geophys. Res. Lett., 29(24), 2181-2184, 2002.

Mayaud, P. N.: Derivation, Meaning, and Use of Geomagnetic Indices, Geophys. Monogr., 22, American Geophysical Union, Washington, D.C., 1980.
McPherron, R. L.: Current status of the growth phase controversy, EOS Trans., AGU, 55, 994, 1974.

McPherron, R. L., Baker, D. N., Bargatze, L. F., Clauer, C. R., and Holzer, R. E.: IMF control of geomagnetic activity, Adv. Space Res., 8, 71-86, 1988.

Newell, P. T., Liou, K., Sotirelis, T., and Meng, C.-I.: Polar UVI observations of global auroral power as a function of polar cap size and magnetotail stretching, J. Geophs. Res., 106, 5895-5905, 2001.

O'Brien, T. P. and McPherron, R. L.: Forcasting the ring current index $D_{s t}$ in real time, J. Atmos. Solar-Terr. Phys., 62, 12951299, 2000.

Opgenoorth, H., Persson, M. A. L., and Olsson, A.: The substorm onset seen with ground-based instrumentation: results, problems, future possibilities, in: Proc. Third International Conference on Substorm (ICS-3), Versailles, France, ESA SP-38, pp. 307-314, 1996.

Sharma, A. S.: Assessing the magnetosphere's nonlinear behavior: Its dimension is low, its predictability, high, Rev. Geophys., 33 , 645-650, doi:10.1029/95RG00495, 1995.

Takalo, J. and Timonen, J.: Neural network prediction of AE data, Geophys. Res. Lett., 24, 2403-2406, 1997.

Temerin, M. and Li, X.: A new model for the prediction of $D_{s t}$ on the basis of the solar wind, J. Geophys. Res., 107, A12, SMP 31, 2002.

Theodoridis, S. and Koutroumbas, K.: Pattern Recognition, 2nd edition, Academic Press, 2003.

Tsyganenko, N. A.: A model of the near magnetosphere with a dawn-dusk asymmetry 2. Parameterization and fitting to observations, J. Geophys. Res., 107, A8, SMP 10-1-SMP 10-17, 2002.

Ukhorskiy, A. Y., Sitnov, M. I., Sharma, A. S., and Papadopoulos, K.: Global and multiscale aspects of magnetosphericdynamics in local-linear filters, J. Geophys. Res., 107(A11), 1369, doi:10.1029/2001JA009160, 2002.

Ukhorskiy, A. Y., Sitnov, M. I., Sharma, A. S., Anderson, B. J., Ohtani, S., and Lui, A. T. Y.: Data-derived forecasting model for relativistic electron density at geosynchronous orbit, Geophys Res. Lett., 31, L09806, doi:10.1029/2004GL019616, 2004.

Valdivia, J. A., Sharma, A. S., and Papadopoulos, K.: Prediction of magnetic storms by nonlinear models, Geophys. Res. Lett., 23, 2899-2892, 1996.

Valdivia, J. A., Vassiliadis, D., Klimas, A., and Sharma, A. S.: Modeling the spatial structure of the high latitude magnetic perturbations and the related current systems, Phys. Plasmas, 6, 41854194, 1999.

Vassiliadis, D., Fung, S. F., and Klimas, A. J.: Solar, interplanetary, and magnetospheric parameters for the radiation belt energetic electron flux, J. Geophys. Res., 110, A04201, doi:10.1029/2004JA010443, 2005.

Vassiliadis, D., Klimas, A. J., Baker, D. N., and Roberts, D. A.: A description of the solar wind-magnetosphere coupling based on nonlinear filters, J. Geophys. Res., 100, 3495-3512, 1995.

Watanabe, M., Iijima, T., Nakagawa, M., Potemra, T. A., Zanetti, L. J., Ohtani, S.-I., and Newell, P. T.: Field-aligned current systems in the magnetospheric ground state, J. Geophys. Res., 103, 68536869, 1998. 